\title{
Listening Effort through Depth of Processing in School-age Children
}

Benson Cheng-Lin Hsu ${ }^{1,2}$, Filiep Vanpoucke ${ }^{2}$, Astrid van Wieringen ${ }^{1}$

${ }^{1}$ Research Group Experimental Oto-Rhino-Laryngology,

Department of Neurosciences, Katholieke Universiteit Leuven, Leuven, Belgium.

${ }^{2}$ Cochlear Technology Centre Belgium, Mechelen, Belgium.

\section{Conflicts of Interest and Source of Funding}

This research was funded by European Commission Marie Curie Actions (FP7-PEOPLE-2013ITN) iCARE Initial Training Network.

Address correspondence to Benson Cheng-Lin Hsu, Research Group ExpORL, Department of Neurosciences, KU Leuven, Herestraat 49 bus 721, 3000 Leuven, Belgium.

Telephone: +32 15795532. E-mail: bhsu@ cochlear.com. 
Objectives: A reliable measure of listening effort is crucial for helping children with communication disorders. In this paper, we propose a novel behavioural paradigm designed to measure listening effort in school-age children and present the results of its validation in a group of normal-hearing children. The paradigm consists of a classic word recognition task in quiet and noise coupled to one of three categorization tasks requiring the children to judge either the color of two pictures or the meaning of the presented words. The response time from the categorization tasks is considered the primary indicator of listening effort.

Design: The listening effort paradigm was evaluated in a group of 31 normal-hearing children 7 to 12 years of age without any history of language or developmental disorders. Custom software was developed to allow administration of the entire paradigm from a conventional laptop computer. A separate touch screen was used as a response interface to gather response time (RT) data from the children. All verbal responses were judged from an experienced tester in order to produce a word recognition score (WRS) for each condition. The software presented randomized lists of 146 selected Dutch nouns in one of three signal-to-noise ratios (SNR) to examine the potential effect of noise on both WRS and RT. Three distinct categorization tasks were also developed to examine how different depths of semantic processing would affect the two outcome measures of WRS and RT. It was hypothesized that, while listening effort as measured by RT would be affected by both noise level and the depth of semantic processing, WRS performance would be affected by changes in noise level only.

Results: There was a significant effect of SNR level on school-age children's WRS: their word recognition performance tended to decrease with increasing background noise level. However, depth of processing did not appear to affect WRS. Moreover, a repeated-measure analysis of variance (rANOVA) fitted to transformed RT data revealed that this measure of listening effort in normal-hearing school-age children was significantly affected by both SNR level and the depth of semantic processing. There was no significant interaction between noise level and the type of categorization task with regard to RT.

Conclusion: The patterns of WRS and RT as observed in our data sets provided support for our general hypothesis regarding the effects of background noise and semantic processing level on speech understanding and listening effort. Even though the magnitude of change in listening effort did not differ between the three novel categorization tasks, the exploratory nature of the current study warranted further evaluation of processing depths as a potential listening effort assessment paradigm in both normal-hearing and hearing-impaired populations. 


\section{Introduction}

Hard-of-hearing persons often describe listening in acoustically challenging environments as demanding and effortful, which can be accompanied by feelings of mental exhaustion at the end of the day (e.g., Hétu et al. 1988; Hornsby 2013). Despite these complaints, common audiological batteries with puretone audiometry and a selection of speech intelligibility tests have remained largely unchanged for more than 60 years (cf. Hirsh et al. 1952) but do not include any clear evaluation of the effort aspect of a patient's hearing experience. Consequently, there has been a recent surge of interest in "listening effort" both as a topic of research and debate among scientists and as a potential outcome indicator of aural rehabilitation among practicing clinicians.

\subsection{Listening Effort}

A brief overview of the theoretical models on human cognitive and perceptual abilities may help us come to a clear working definition of 'listening effort'. In his seminal work, Kahneman (1973) argued that there is a limited supply of cognitive capacity for an individual to perform any kinds of mental work, even if he/she is generally free to divide this limited capacity among concurrent tasks (Moray 1967). More recently, the Ease of Language Understanding (ELU) model (Rönnberg et al. 2013) posits that incoming linguistic signals are rapidly, automatically, and multimodally bound together with pre-existing phonological representations in semantic long-term memory speech recognition, resulting in relatively effortless speech understanding. The ELU model refers to this implicit process as a "match". However, a "mismatch" with phonological representations can also occur when language inputs are degraded by competing signals or hearing impairment. The "mismatch" scenario necessitates slower, more laborious language processing 'loops' to fill in missing linguistic information until a desired level of understanding is reached. This top-down explicit process consumes working memory capacity, increases cognitive load, and consequently decreases the subjective 'ease' of listening.

Given the theoretical models above, a common way to understand listening effort is the expenditure of limited cognitive resources (such as working memory capacity) in order to successfully understand incoming speech signals (Hicks \& Tharpe 2002; Gosselin \& Gagne 2010; Gustafson et al. 2014). Despite increased research activities in this area, the wider academic community has yet to agree on the most optimal way(s) of measuring listening effort (McGarrigle et al. 2014). A variety of techniques found in the literature can be broadly categorized into three groups: 1) subjective methods, such as the use of a standardized Speech, Spatial, and Qualities of Hearing Scale (Akeroyd et al. 2014) or a Visual Analog Scale tailored for listening effort (e.g., Rudner et al. 2012); 2) behavioural methods, such as repeating sentences while visually tracking a moving dot (Desjardins \& Doherty 2014) or monitoring running speech for substituted words (MacPherson \& Akeroyd 2013); 3) physiological methods, such as measuring skin conductance (Mackersie \& Cones 2011) or the extent of pupil dilation (Zekveld et al. 2010). Compared to interviews or questionnaires, behavioural paradigms aim to make listening effort measurable without evoking the subjects' own judgment, which is often affected by both intrinsic and extrinsic factors that are hard to control. Furthermore, behavioural paradigms do not usually call for sophisticated skill-intensive equipment like most physiological methods do, which is an advantage that increases the former approach's clinical feasibility. Two excellent review articles by Gosselin and Gagne 
(2010) and McGarrigle et al. (2014) provide comprehensive overviews of the various approaches that have been attempted when it comes to measuring listening effort.

Since the cognitive resources available for 'listening' are limited, a behavioural experiment can be designed to measure 'effort' by introducing an additional task that competes for the scarce resources. This is often referred to as a "dual-task paradigm", where the primary task involves listening and repeating speech materials and the secondary task is an activity that further taxes the system (Gosselin \& Gagne 2010). If one makes the listening task more difficult through, for example, the addition of background noise, then more cognitive capacity would be required to maintain an acceptable level of speech recognition (Moray 1967) and thereby decreasing the supply of resources that is available to perform the secondary task. One method of quantifying this said performance is by measuring the reaction or response time it takes to complete the secondary task (e.g., Hicks \& Tharpe 2002; Sarampalis et al. 2009; Houben et al. 2013; Pals et al. 2013; Picou \& Ricketts 2014b). A lengthening of reaction/response time in the same participant is indicative of an increase of cognitive resource consumption by the primary listening task and by extension an increase in the level of listening effort.

\subsection{Depth of Processing}

A recent study by Picou and Ricketts (2014b) has shown that changes in listening effort as measured by reaction time between Yes-noise and No-noise conditions in a behavioural dual-task experiment were significantly greater when the secondary task involved judging the grammatical property of a word than when the secondary task was a visual task unrelated to the word. This effect was demonstrated in both normal-hearing and hard-of-hearing adults. The authors offered two possible rationales for their findings: 1) there was increased resource competition in the same modality domain due to both primary and secondary tasks being auditory in nature, and 2) the secondary grammatical task involved a deeper level of processing than either the simple or complex visual task and thereby placed a higher demand on the limited overall cognitive capacity of their research participants.

The "depths of processing" framework was first described in Craik and Lockhart (1972). The framework argues that sensory inputs, regardless of modalities, are processed in a hierarchical fashion. For example, to perceive a word auditorily, a shallow level of processing would be the rapid analysis of acoustic characteristics such as pitch or rhythm whereas deeper levels of processing would concern mapping syllabic information to pre-existing patterns (see the definition of a "match" in the section on the ELU model above) and the discernment of meaning and associations from the same word. Craik and Lockhart (1972) further suggest that one of the 'byproducts' of processing stimuli at a deeper level is longer information retention due to more reinforced 'memory traces'. This is supported by more recent studies such as Rudner et al. (2013), which reports better working memory performance when information is processed at a semantic (deeper) level compared to a phonological (shallower) level. According to the depth-of-processing framework, once a word is recognized by a listener, the speech stimulus may undergo further enrichment or elaboration by being associated with elements of the listener's past experience. This phenomenon of extensive mental analysis at the terminal stage of perceptual processes has been termed the "elaboration of processing" (Eysenck \& Eysenck 1979). There is evidence that a deep level of processing combined with a high degree of elaboration could lead to 
even better memory test performance compared to the same depth of processing with little or no elaboration (Craik 1977).

Our discussions above may be distilled into three concepts relevant to assessing listening effort: 1) the total human cognitive capacity is ultimately limited (Kahneman 1973); 2) noise-degraded speech signals tax the cognitive capacity by requiring more overt processing according to the ELU model (Rönnberg et al. 2013); 3) a deeper level of processing further increases the cognitive load of the listener (Eysenck \& Eysenck 1979). By inference, changes in listening effort in a dual-task paradigm may become more susceptible to the effect of background noise if one increases the depth of processing required by the secondary task. Indeed, this is what Picou and Ricketts (2014b) have found. Moreover, if greater elaboration of processing leads to better retention and stronger memory traces due to increased resource consumption compared to a deep-processing task without elaboration, then a dual-task assessment requiring a greater level of semantic elaboration would be even more affected by noise and would therefore be more sensitive when investigating the effects of noise on listening effort. We strive to test these hypotheses through the design of our behavioural paradigm in the current study.

\subsection{School-age Children}

The intellectual development of children during their formative elementary school years is facilitated by an easy access to spoken language in a classroom setting. However, not only has excessive noise in the school been identified as a risk factor for childhood fatigue and headache (Wålinder et al. 2007), prolonged periods effortful learning (Downs \& Crum 1978) or mental fatigue (Bess \& Hornsby 2014) may also exert deleterious effects on the educational outcomes of hard-of-hearing children. This issue is gaining importance by the increasing placement of students with severe to profound hearing loss who are otherwise healthy in mainstream schools (Venail et al. 2010) as cochlear implantation becomes widely accepted as a standard treatment over the last two decades (Kral \& O'Donoghue 2010). The difficulties these children experience are well documented in the literature (e.g., Preisler et al. 2005; van Wieringen \& Wouters 2015).

Since listening effort is a new field of research, most of the available literature includes only adult subjects likely due to the need to validate novel methods in a population that is relatively easy to both access and test. Indeed, there are only a handful of published studies on listening effort with teen-age or pediatric participants (e.g., Choi et al. 2008; Howard et al. 2010; Hughes \& Galvin 2013; Gustafson et al. 2014). Moreover, since children experience significant linguistic and cognitive maturation during their elementary school years (e.g., Cartwright 2002; Piaget \& Inhelder 2008), the same behavioural paradigm may yield different patterns of results between children and adults. Given the concerns over the negative impacts of chronic mental fatigue in a mainstream-school environment, listening effort in school-age children is a critical knowledge gap that warrants more research attention.

\subsection{Purpose}

The objective of the present study is to investigate changes in listening effort due to changes in background noise level and changes in the depth and elaboration of processing in a group of normalhearing school-age children. We intend to achieve this aim through a behavioural paradigm that utilizes 
a conventional word recognition task combined with one of three novel categorization tasks designed to tap into three different stages of speech stimulus processing. The speed of response with which a child completes a categorization task (i.e., the response time) will be the primary measure of listening effort in the current paradigm. It is hypothesized that increasing degradation of speech signals through background noise would lead to decreasing word recognition performance. Listening effort is hypothesized to increase with increasing speech degradation, and the magnitude of change in listening effort would differ between categorization tasks due to different levels of processing involved. Increasing the depth or elaboration of processing in itself is also expected to increase listening effort but only have minimal, if any, impact on word recognition scores in school-age children.

\section{Materials and Methods}

\subsection{Participants}

A priori power analysis suggested that, for a medium effect size Cohen's $f$ of 0.25 , at least 27 subjects were necessary to achieve a minimum power level of 0.80 in a repeated-measure design with 9 unique conditions where the interaction between factors was to be investigated. With this in mind, we recruited 31 normal-hearing children age 7 to 12 years old (mean $=10.0$ years, SD $=1.3$ ) for the study. Each child was registered as a student at a regular Dutch/Flemish elementary school with no reported history of language or developmental disorders. Standard pure-tone audiometry was performed on each child at the beginning of each experimental session to ensure bilateral normal hearing $(<15 \mathrm{~dB} \mathrm{HL})$ in the prominent speech region $(500$ to $4000 \mathrm{~Hz}$ ). The receptive language abilities of each child were also assessed through two sub-tests from the Dutch-version Clinical Evaluation of Language Fundamentals (CELF-4-NL): Word Classes - Receptive ("WCR") and Understanding and Following Directions (shortened to "BAV" in Dutch).

A parent of each participant signed a letter of consent explaining the purposes and procedures of the study. Direct verbal assent was also obtained from the child after explaining that they were free to terminate participation at any time without any consequences. Each child was rewarded 10 euros as a token of gratitude for their participation. The medical ethical committee of UZ Leuven/KU Leuven approved the participant selection criteria and test procedures (Approval No. B322201523779).

\subsection{Test Materials}

Brainstorming among the three authors resulted in an initial list of over 200 candidate Dutch nouns deemed suitable for the construction of the current paradigm. Since our semantic categorization tasks required children to judge if a word referred to an animal or something dangerous, target words were selected to represent a fair distribution among the four categories of harmless animals, harmless nonanimals, dangerous animals, and dangerous non-animals. The word pool was then narrowed down to 159 entries by keeping candidate words only if they were also listed in a published reference book (Schaerlaekens et al. 1999) of standard Dutch vocabulary known by native Flemish and Dutch children age 5 to 6 years old. The validity of these 159 words was further verified by surveying 14 children age 6 to 12 years who were native speakers of Dutch. For each word, the questionnaire first asked the child "Do you know this word", and if the answer was "Yes", then the two follow-up questions were "Is this an 
animal" and "Is this something dangerous". Children either completed the questionnaire alone or jointly with their parents. Thirteen of the words were subsequently omitted either because a word was known by less than 9 of the 14 children ( $<64 \%$ knowledge rate) or because a word had more than one salient meanings that belonged in different semantic categories (e.g., "tank"). The final pool thus consisted of a total of 146 Dutch nouns. Table 1 details the percentage distribution of word syllable count in each of the four semantic categories.

A professional female speaker produced the words. Digital recordings were made in a double-walled sound-attenuating booth using a Sennheiser HS-2 microphone and an Edirol R-4 PRO sound card (Roland). Several tokens of the same word were articulated, and the most clearly pronounced one was selected. Each recording was segmented in Praat (Boersma \& Weenink 2013) to extract individual words, which were then stored in a waveform audio file format (wav) at $44.1 \mathrm{kHz}$. Speech-weighted stationary noise based on the long-term average speech spectrum of the full speech corpus was developed. The use of a spectrally matched masker ensures that, on average, the signal-to-noise ratio will be approximately equal at all frequencies. Further waveform analysis carried out in Matlab suggested that all recordings had a standard deviation of 2-3 dB in speech loudness variations.

\subsection{Signal-to-Noise Ratios}

All test word sound files were presented at a fixed level of $65 \mathrm{~dB}$ SPL throughout the experiment. The custom software achieved three signal-to-noise ratio (SNR) conditions by varying the level of the speechweighted noise: "Quiet», "O SNR», and «-5 SNR». The noise, if selected, started three seconds before the first word was presented and remained on at a constant level for the entire duration of a trial run. These noise conditions were chosen to represent differing degrees of signal degradation as well as to mimic typical classroom SNRs, which were reported to vary between +5 to $-7 \mathrm{~dB}$ (Crandell \& Smaldino 1994; Howard et al. 2010). Moreover, pilot testing with a small group of children suggested that the repetition accuracy at an SNR of $+5 \mathrm{~dB}$ was not significantly different from that in the quiet condition, showing a ceiling effect in normal-hearing children. As a result, the «+5 SNR» condition was replaced by a more difficult «-5 SNR» condition.

\subsection{Categorization Tasks}

The cognitive component of the listening effort assessment consisted of three categorization tasks/conditions that were designed to manipulate the level of semantic processing a child would need to perform after given a chance to hear a target word.

The first task, "Color», was a visual judgment task where the distinction was between two identical pictures, one black-and-white and the other vibrantly colored with many hues. The two pictures were presented at the same time on the touch screen, where the colored one had an overall $50 \%$ chance of appearing on either the right or left side. The participants were instructed to 'touch' the colored picture as soon as it appeared on the screen. The "Color» task utilized visual stimuli that were wholly unrelated to any of the test words and thus required little in the way of semantic processing. 
The second categorization condition, "Animal», was an auditory word classification task where a child had to indicate if a spoken word represented an animal or not. The response interface of this condition was very similar to "Color» in that there were also two 'buttons' on the screen (one meaning "Yesanimal" and the other "No-animal") except that the position of the buttons stayed constant throughout the condition. In the original study by Picou and Ricketts (2014b), one experimental condition asked young adults to distinguish if a word was a noun or not. In our opinion, a grammatical exercise such as this would be too challenging for the majority of primary-school students. The «Animal» distinction involved a familiar and mildly interesting subject more appropriate for our target age group. The "Animal» task was designed so that each target word had to be processed deeply at the semantic level by the children unlike the aforementioned «Color» task, where no more processing was required beyond the mere recognition of a word.

The last categorization condition, «Dangerous», was procedurally similar to «Animal» except that the «Dangerous» task asked children if a target word they heard represented something potentially dangerous or harmful. Among the three, the «Dangerous» condition was the one that required the deepest level of semantic processing in the form of elaboration and imagination based on the meaning of a test word. Since there is evidence that young children around 4 to 5 years of age can already grasp the basic concept of 'danger' (Hill et al. 2000), the "Dangerous» categorization was also deemed appropriate for children enrolled in mainstream elementary schools. Even though both «Dangerous» and "Animal» were auditory-only categorization tasks requiring processing at the semantic level, the question "Is this something dangerous?" was expected to invoke greater processing elaboration and stimulus enrichment (Craik \& Lockhart 1972) through extensive imagination and association with past experience than the relatively simpler question "Is this an animal?".

\subsection{Software and Equipment}

The custom software used in the study was developed in the C\# language under the .NET framework for a Windows computer. Its main screen allowed a tester to enter relevant data as well as to specify condition parameters, while its subsidiary screen was projected to a touch screen sitting directly in front of a participant. A high-precision timer was built into the software to ensure high accuracy and reliability when measuring response times within a Windows operating environment. In-house testing showed that this timer yielded an error margin of less than 10 micro-seconds when compared to system BIOS values. Upon running the program, the software would select 15 words from the pool of 146 words according to pre-defined rules and set them aside as training materials. Then, nine semi-random word lists consisting of 30 words each would be generated for use in each of the nine (3 SNRs $x 3$ categorizations) conditions according to pre-defined distributions of non/animal and non/dangerous words. No words could ever appear more than once in each of the categorization tasks to minimize any training effect. Moreover, words with the same stem (e.g., the stem "bear" in words such as "ice bear" and "panda bear") could never appear together in the same list to avoid confusion. The order of words in each list (including the training list) was automatically randomized each time the word list was selected and played. 
The software was installed on Windows 8.1 laptop computers connected to a 10-inch external portable touch screen (GeChic On-Lap 1002), which served as the visual display and response input device for the participants. A dedicated verification test suggested that the touch screen randomly added between $<1$ to $20 \mathrm{~ms}$ to final response time measurements, which was within the latency value specified by the manufacturer. The Dutch words were routed through an external sound card (RME Fireface UC) and presented through supra-aural headphones (Sennheiser TDH 200) binaurally. The stationary speechweighted noise was used to calibrate the headphones at $65 \mathrm{~dB}$ SPL, measured through a clamped-down artificial ear (Type 4153, Brüell \& Kjær) connected to a sound level meter prior to data collection.

\subsection{Test Procedures}

All testing was carried out in a quiet room inside the child's own home. This location was chosen to achieve the balance between minimizing unwanted distractions and testing in a familiar environment where children would feel most at ease. The parents were requested to reserve a quiet, undisturbed location inside the home for a minimum duration of one hour before any testing took place. The child and the tester were seated at a 90-degree angle to each other, and all equipment was arranged on the same tabletop surface in such a way that the child would not be able to see the tester's laptop screen. The touch screen was placed directly facing the child. Between the child and the touch screen was a piece of laminated paper with markings indicating where the child should put their dominant hand (either left or right) and where the touch screen should align. The distance between the screen and the fingers was approximately $10 \mathrm{~cm}$.

The entire experiment was designed to be completed within one appointment of approximately 60 minutes. A session would always begin with the administering of pure-tone audiometry and the CELF-4NL language tests. A short break for each child was scheduled between these pre-tests and the rest of the experiment to control for the number of interruptions. Then, instructions for the experiment would be conveyed verbally until the child fully understood the nature of the tasks. Practice runs with the training materials would be carried out to familiarize the child to each of the three categorization conditions. Figure 1 summarizes the current behavioural paradigm and provides more detail regarding the interaction between the participant, the tester, and the software at each trial of the experiment. The order of the test conditions was systematically counterbalanced as an additional guard against training effect. The recording of the response time during the categorization tasks was automatically handled by the custom software: a valid measurement was defined as the elapsed time in millisecond between the end of a sound file and the first instance a child touched the screen to indicate a categorization response. The correctness of verbal repetitions, on the other hand, was judged by the tester: a correct repetition was defined as whenever a child's utterance matched a word transcription shown on the laptop screen to the best of the tester's knowledge. Any non-response 10 seconds after the word presentation would be marked as "No Response" (NR) for the categorization task and "Wrong" for the verbal repetition task. The overall pace of a trial run was controlled by the tester as the software

was programmed to wait for the verbal repetition correctness input from the tester before advancing to the next word. At the completion of each 30-word trial run, the software displayed the percentage of correct repetitions as well as the average response time on the tester's screen and stored all collected data in the form of a Microsoft Excel file. 


\section{Results}

The «Color» categorization was a binary-choice task with 30 tokens in each trial run. Chance analysis incorporating these parameters indicated that one had to obtain a correctness score higher than $65 \%$ to be considered significantly $(p<0.05)$ above a "guessing" performance level. Upon close examination of the data, one of the participants (age $=8.6$ years) did not meet this criterion under all three SNR levels of the "Color» task, raising considerable doubt into whether test instructions were fully grasped or not. All data from this particular child were subsequently excluded from further analysis, leaving the final number of analyzed data sets to $n=30$. This also suggested that the vast majority of our school-age participants (96.8\%) had successfully grasped the instructions of the listening effort assessment.

\subsection{Word Recognition Score}

A Word Recognition Score (WRS) was calculated for each condition for each child by taking the percentage of correct verbal repetitions out of a list of 30 words. Table $\mathbf{2}$ provides an overview of the median WRS and associated interquartile ranges for all of the nine conditions. Examination of the WRS data reveals a clear ceiling repetition performance at all three of the "Quiet» conditions. Since the Shapiro-Wilk test indicated that the majority of condition-wise WRS was not normally distributed (especially the "Quiet» conditions due to the ceiling effect), we employed two separate non-parametric Friedman tests to evaluate the relationship between WRS and the two independent factors: "SNR" ("Quiet», "0 SNR», "-5 SNR») and "Task" («Color», «Animal», "Dangerous»). The results indicated that, while "SNR" was a statistically significant factor when the WRS values were averaged across "Task", $\chi 2(2)=154.81, p<0.001$, "Task" was not found to be significant when averaging WRS across "SNR". As shown in Table 2, post hoc analysis with the non-parametric Wilcoxon signed-rank test showed that noise conditions in all three possible pairs («Quiet» vs. "0 SNR», "Quiet» vs. «-5 SNR», "0 SNR» vs. «-5 SNR») were all significantly different from each other ( $p<0.001$, Bonferroni correction applied). In other words, even though the WRS measure was negatively affected by increasing noise levels, it was not significantly affected by the secondary categorization tasks in each SNR level.

\subsection{Response Time}

The primary measure of listening effort in the current behavioural paradigm was the elapsed time between the end of a target word presentation and the first moment the screen was 'touched' to indicate a categorization response. A mean Response Time (RT) measure was prepared for further analysis by averaging trial-level response time records (while excluding the "No Response" entries) for each condition for each child. Figure 2 summarizes the resultant RT data set across the nine $(3 \times 3)$ experimental conditions.

The question of whether to adjust the RT data for repetition or categorization correctness warrants several points of consideration. Since the "Color» task was visual in nature and unrelated to the auditory stimulus, it did not appear logical to adjust the RT data from «Color» for verbal repetition correctness. Moreover, analysis of the WRS data as presented above suggested that instances of incorrect repetition increased systematically with background noise level. In other words, mishearing words could be thought of as a natural and expected 'symptom' of challenging listening situations. We believe, 
therefore, including RT data from both correct and incorrect verbal repetitions would result in better representation of the varying levels of listening effort people experience in real-life situations. As for categorization correctness, Table 3 indicated that there were very few instances $(0.6 \%$ to $1.3 \%)$ where the wrong choice was selected during "Color» across all SNR conditions. Hence, the effect of adjusting RT for correct categorization could be expected to be negligible for the «Color» condition. Moreover, sometimes an apparent 'wrong' categorization could be due to a misheard word. For example, during "Animal», if a child misunderstood the word bijl ("axe" in Dutch) as bij ("bee" in Dutch), his/her judgment of "Yes-animal" would in fact be correct based on the perceived word but be marked as "wrong" based on the pre-determined categories stored in the software. In this instance, no more and no less cognitive capacity would be spent to process the meaning than if the correct word was perceived in the first place. Finally, Table 3 suggested that, while mean categorization error rates differed between "Animal» and "Dangerous» when children repeated words correctly, they were in fact similar when children also made repetition mistakes. These results were to be expected, since the "Dangerous» task was designed to encourage imagination and semantic association in such a way that participant responses would be invariably shaped by their preconceptions and experiences. Therefore, adjusting the RT data sets for categorization correctness would not make sense given the nature of our semanticprocessing conditions, especially «Dangerous».

A repeated-measure analysis of variance ( $\mathrm{rANOVA}$ ) was selected as the underlying statistical model for the RT measurements. However, preliminary analysis with histograms and the Shapiro-Wilk test suggested that the RT data set did not conform to a Gaussian normal distribution, which was a crucial assumption when fitting a model such as rANOVA. To remedy this issue, a Johnson's transformation (Johnson 1949) was applied to make the RT data normal. Afterwards, the rANOVA was fitted with two specified within-subject factors, each with three levels, according to the experimental design of our paradigm: "SNR" («Quiet», «O SNR», «-5 SNR») and "Task" («Color», «Animal», «Dangerous»). Additionally, a between-subject factor, "Participant", was also specified for the model to account for the fact that the effects of changing background noise and semantic processing levels might be different from child to child. Mauchly's Test of Sphericity demonstrated that the assumption of sphericity had not been violated for both within-subject factors. The results indicated a significant main effect of "SNR", $F(2,58)=85.81, p<0.001$, as well as "Task", $F(2,58)=112.84, p<0.001$. However, there was no significant interaction between "SNR" and "Task" with the transformed RT data.

Pairwise post hoc analysis of the non-transformed RT data with Bonferroni correction revealed the significance of RT changes between the three SNR conditions used in the experiment. For "O SNR», normal-hearing children's RT on average increased by $102 \mathrm{~ms}(p=0.014)$ when compared to the "Quiet» condition. For «-5 SNR», RT on average increased by $351 \mathrm{~ms}(p<0.001)$ compared to «Quiet». Between «0 SNR» and «-5 SNR», the average RT tended to increase by $249 \mathrm{~ms}(p<0.001)$ when background noise level was increased by $5 \mathrm{~dB}$ SPL. Likewise, significant RT changes were also found when the data from the three categorization tasks were compared in a pairwise fashion with Bonferroni correction. For «Animal», normal-hearing children's RT on average increased by $321 \mathrm{~ms}(p<0.001)$ when compared to the shallow-processing «Color» task. For «Dangerous», RT on average increased by $480 \mathrm{~ms}(p<0.001)$ compared to «Color». Between «Animal» and «Dangerous», the average RT tended 
to increase by $159 \mathrm{~ms}(p=0.003)$ when deep semantic processing demanded even more mental elaboration and stimulus enrichment from our school-age children.

\section{Discussion}

\subsection{Changes in Word Recognition}

The analysis of the WRS data indicated that the performance of normal-hearing school-age children on the repetition task was significantly affected by both the addition and increase of background noise. This is expected, as speech-shaped noise energetically masks parts of the speech signals and consequently makes accurate deciphering of them more difficult. Conversely, our participants did not exhibit different word recognition performance pattern with different categorization tasks. Since the children were instructed to perform the actions of listening, categorizing, and repeating sequentially, there were no immediate reasons to suspect "task interference" from having to perform the repetition and categorization tasks simultaneously. However, the present paradigm did artificially insert a short gap between the perception and the repetition of target words to 'make room' for the categorization task. This raises the possibility that different categorization tasks could influence the memory trace of a perceived target word still fresh in a child's mind. Given that past studies such as Craik and Lockhart (1972) and Craik (1977) found better information retention due to better reinforced memory traces from processing speech signals at a deeper or more elaborate level, one could argue that deep semantic conditions such as "Animal» or "Dangerous» would result in children remembering the words better (and therefore having better recognition scores) vis-à-vis non-semantic conditions such as «Color». Nevertheless, since only brief (typically less than 2 seconds; see Figure 2) retention of single, familiar words was ever required and observed from the participants, our paradigm was most likely not sensitive enough to detect any incidental memory benefit from increasing the depth or elaboration level of verbal processing. For these reasons, we hypothesized that different categorization tasks would not alter school-age children's WRS in any significant way, and this was indeed what our data had shown.

\subsection{Changes in Listening Effort}

The analysis of the RT data indicated that average listening effort in normal-hearing school-age children tended to rise with background noise level when measured by each one of the categorization tasks. This finding was consistent with the detrimental effect of noise on effort-related measures reported in previous studies (e.g., Downs \& Crum 1978; Picou et al. 2013; Pals et al. 2015). Howard et al. (2010) also echoed our result by demonstrating that a more challenging SNR was correlated with poorer digit recall performance in school-age children when compared to a more favourable SNR. Furthermore, demands on cognitive capacity, as indicated by the patterns of RT, also increased when we changed the categorization task from one that required only shallow semantic processing ("Color») to one that demanded deep semantic processing («Animal»). Cognitive demands further increased when the categorization condition changed from one that involved a 'simpler' form of deep semantic processing («Animal») to one that required additional elaboration and enrichment of the perceived speech signal («Dangerous»). This result not only provided support for our hypothesis about the effect of changing the 
depth and elaboration level of semantic processing but also validated the three novel categorization tasks in the current paradigm as appropriate 'probes' for three desired levels of processing.

The lack of significant interaction between the two independent variables "SNR" and "Task" observed in the RT data sets was contrary to our hypothesis and suggested that the effect of background noise on the RT measure did not differ across different categorization conditions. In other words, relative to the average RT from «Color», neither «Animal» nor «Dangerous» appeared to increase the listening effort measure at «-5 SNR» more than what they did at either «Quiet» or "0 SNR». If deep-processing tasks did not make the test paradigm more 'sensitive' to the effects of noise, then perhaps the alternative "Color» task would be preferred for its relative simplicity and smaller outcome measure variability (see the SD numbers presented in Figure 2) when assessing listening effort in a clinical setting. Such speculation would stand in clear contrast to the conclusion reached in Picou and Ricketts (2014b). In their study, the greatest effect of noise on the measure of listening effort took place with the secondary task that demanded the deepest level of semantic processing from both of their normal-hearing and hard-of-hearing adult participants. There are two potentially relevant methodological differences when one considers the discrepancy in findings between the two studies. Firstly, the speech materials in Picou and Ricketts (2014b) were presented at 'floating' SNRs based on a pre-defined performance level, whereas the target words in the current study were presented at fixed SNR levels. While fixed presentation levels may have the advantage of being more realistic (e.g., different children in the same classroom would generally be exposed to the same instantaneous SNR), they also allow performance ceiling or flooring to happen to some of the children that may in turn obscure any potential interaction effect. Secondly and building on Kahneman's (1973) original model of attention and limited cognitive capacity, the latest theoretical framework of listening effort (Pichora-Fuller et al. 2016) incorporates "motivation" as an important predictor modulating the level of effortful listening one may experience at a given moment. It is possible that many school-age children might have treated a multi-task test of listening effort as a mildly entertaining 'game', whereas adults would tend to approach a similar paradigm as some sort of 'duty' depending on how they became involved with the study. This subtle difference in motivation might have affected the amount of general effort exerted by different age groups and could also influence their respective perception of listening effort (Picou \& Ricketts 2014a). In short, these considerations point to the need for future studies to examine how methodological differences (e.g., fixed vs. adaptive SNR) and participant traits (e.g., underlying motivation) could affect a listening effort measure such as RT.

Thinking from a broader perspective, the lack of significant interaction between the two manipulated variables "SNR" and "Task" in terms of RT and the fact that both WRS and RT were significantly affected by an increase in background noise might cast some doubt on the validity of RT as an effective measure of listening effort. While this genuinely points to a potential weakness of the novel behavioural paradigm proposed in the current paper, the relatively small sample size $(n=30)$ and the exploratory nature of our study preclude any firm conclusion to be drawn at this point. The patterns of WRS as well as RT as observed from our data sets suggest that the full potential of a listening effort assessment built around the concept of depth of processing is yet to be discovered through continued, rigorous research effort in this crucial field. 
Lastly, the pattern of RT changes from the «Color» condition also warrants some thoughts. According to the three-component theory of working memory (Baddeley \& Hitch 1974; Baddeley 2003), a separate pool of cognitive resources is available for each of the two storage systems of working memory: the "visuospatial sketchpad" and the "phonological loop". Since "Color» involved visual stimuli that were wholly unrelated to the target words (and therefore both working memory sub-systems should be activated), one would expect the performance on the visual distinction task would be independent from any increase in resource demand in the auditory domain. However, our data showed that the mean RT from all three categorization conditions, including «Color», increased proportionally as background noise level increased. This finding suggests that the exact underlying mechanisms of behavioural paradigms involving multi-modal inputs and multi-level processing may be more complex than at first glance, and more research effort is undoubtedly needed before stronger links can be established between listening effort and existing theories of cognitive resource structure. Naturally, the observation that the presence of background noise also affects the performance on a simple visual task has tremendous implication for classroom learning where information is delivered auditorily as well as visually. It may be beneficial for future studies to further explore this phenomenon through dual-task designs involving more real-life visual activities performed in a variety of background noise.

\subsection{Conclusion}

The current paper detailed the methods and findings from the testing of a novel behavioural paradigm of listening effort in a group of normal-hearing school-age children. The main task of this paradigm asked the children to listen and repeat auditorily presented target words, while the competing task consisted of one of three categorization 'games' that manipulated the level of required semantic processing. The results suggested that degrading the input signals by means of background noise affected performance on the word recognition task as well as increased listening effort as measured by the time it took for the children to complete each of the categorization tasks. Increasing the depth and elaboration level of word processing also significantly increased listening effort but did not affect the children's repetition performance. Although the data sets derived from our paradigm could not differentiate the three categorization tasks by their sensitivity to changes in listening effort caused by changes in noise level, the overall findings from the present study pointed to future research direction that could help us further understand the interplay between listening effort, depths of semantic processing, hearing impairment, and learning in school-age children.

\section{Acknowledgements}

We are very grateful to Wivine Decoster (KU Leuven), who was the voice behind the recording of the speech materials, Stefan Lievens (Cochlear Technology Centre), who developed the custom software that hosted the behavioural paradigm, and Tobias Busch, who provided invaluable advice regarding suitable statistical methods for the present study. Special thanks go to KU Leuven master's students, Cara Hermans and Inte Bernaerts, who were indispensable during the data collection process.

Each of the three authors contributed significantly to this work: Benson Cheng-Lin Hsu designed and implemented the experiment, analyzed the data, and wrote the paper; both Filiep Vanpoucke and Astrid 
van Wieringen provided close scientific supervision, logistical and statistical support, as well as critical revision throughout the study and the drafting of the manuscript.

This work was supported by the European Commission through the Marie Curie Actions (FP7-PEOPLE2013-ITN) “iCARE” Initial Training Network.

Part of the work was presented at the 12th Congress of the European Federation of Audiology Societies, Istanbul, Turkey, May 29, 2015 and at the 2015 B-Audio Meeting, Namur, Belgium, October 23, 2015.

The authors declare no other conflict of interest.

Address for correspondence: Benson Cheng-Lin Hsu, Research Group ExpORL, Department of Neurosciences, KU Leuven, Herestraat 49 bus 721, 3000 Leuven, Belgium. Telephone: +32 15795532. Email: bhsu@cochlear.com.

\section{References}

Akeroyd, M. A., Guy, F. H., Harrison, D. L., \& Suller, S. L. (2014). A factor analysis of the SSQ (Speech, Spatial, and Qualities of Hearing Scale). International Journal of Audiology, 53(2), 101-114.

Baddeley, A. (2003). Working memory: looking back and looking forward. Nature Reviews Neuroscience, 4(10), 829-839.

Baddeley, A. \& Hitch, G. J. (1974). Working memory. In G. A. Bower (Ed.), Recent Advances in Learning and Motivation, 8. New York, NY: Academic Press.

Bess, F. H., \& Hornsby, B. W. (2014). Commentary: Listening can be exhausting-Fatigue in children and adults with hearing loss. Ear and Hearing, 35(6), 592-599.

Boersma, P., \& Weenink, D. (2013). Praat software. Amsterdam: University of Amsterdam.

Cartwright, K. B. (2002). Cognitive development and reading: The relation of reading-specific multiple classification skill to reading comprehension in elementary school children. Journal of Educational Psychology, 94(1), 56.

Choi, S., Lotto, A., Lewis, D., Hoover, B., \& Stelmachowicz, P. (2008). Attentional modulation of word recognition by children in a dual-task paradigm. Journal of Speech, Language, and Hearing Research, 51(4), 1042-1054.

Craik, F. I. (1977). Depth of processing in recall and recognition. In S. Dornic (Ed.), Attention and Performance VI. Hillsdale, NJ: Lawrence Erlbaum Associates Inc.

Craik, F. I., \& Lockhart, R. S. (1972). Levels of processing: A framework for memory research. Journal of Verbal Learning and Verbal Behavior, 11(6), 671-684.

Crandell, C. C., \& Smaldino, J. J. (1994). An Update of Classroom Acoustics for Children with Hearing Impairment. Volta Review, 96(4), 291-306. 
Desjardins, J. L., \& Doherty, K. A. (2014). The effect of hearing aid noise reduction on listening effort in hearing-impaired adults. Ear and Hearing, 35(6), 600-610.

Downs, D. W., \& Crum, M. A. (1978). Processing demands during auditory learning under degraded listening conditions. Journal of Speech, Language, and Hearing Research, 21(4), 702-714.

Eysenck, M. W., \& Eysenck, M. C. (1979). Processing depth, elaboration of encoding, memory stores, and expended processing capacity. Journal of Experimental Psychology: Human Learning and Memory, 5(5), 472-484.

Gosselin, A. P., \& Gagne, J.-P. (2010). Use of a Dual-Task Paradigm to Measure Listening Effort. Canadian Journal of Speech-Language Pathology and Audiology, 34, 43-51.

Gustafson, S., McCreery, R., Hoover, B., Kopun, J. G., \& Stelmachowicz, P. (2014). Listening effort and perceived clarity for normal hearing children with the use of digital noise reduction. Ear and Hearing, 35(2), 183-194.

Hétu, R., Riverin, L., Lalande, N., Getty, L., \& St-Cyr, C. (1988). Qualitative analysis of the handicap associated with occupational hearing loss. British Journal of Audiology, 22(4), 251-264.

Hicks, C. B., \& Tharpe, A. M. (2002). Listening effort and fatigue in school-age children with and without hearing loss. Journal of Speech, Language, and Hearing Research, 45(3), 573-584.

Hill, R., Lewis, V., \& Dunbar, G. (2000). Young children's concepts of danger. British Journal of Developmental Psychology, 18(1), 103-119.

Hirsh, I. J., Davis, H., Silverman, S. R., Reynolds, E. G., Eldert, E., \& Benson, R. W. (1952). Development of materials for speech audiometry. Journal of Speech and Hearing Disorders, 17(3), 321-337.

Hornsby, B. W. (2013). The effects of hearing aid use on listening effort and mental fatigue associated with sustained speech processing demands. Ear and Hearing, 34(5), 523-534.

Houben, R., van Doorn-Bierman, M., \& Dreschler, W. A. (2013). Using response time to speech as a measure for listening effort. International Journal of Audiology, 52(11), 753-761.

Howard, C. S., Munro, K. J., \& Plack, C. J. (2010). Listening effort at signal-to-noise ratios that are typical of the school classroom. International Journal of Audiology, 49(12), 928-932.

Hughes, K. C., \& Galvin, K. L. (2013). Measuring listening effort expended by adolescents and young adults with unilateral or bilateral cochlear implants or normal hearing. Cochlear Implants International, 14(3), 121-129.

Johnson, N. L. (1949). Systems of frequency curves generated by methods of translation. Biometrika, 36(1/2), 149-176.

Kahneman, D. (1973). Attention and Effort. Englewood Cliffs, NJ: Prentice-Hall. 
Kral, A., \& O'Donoghue, G. M. (2010). Profound deafness in childhood. New England Journal of Medicine, 363(15), 1438-1450.

Mackersie, C. L., \& Cones, H. (2011). Subjective and psychophysiological indices of listening effort in a competing-talker task. Journal of the American Academy of Audiology, 22(2), 113-122.

MacPherson, A., \& Akeroyd, M. A. (2013, June). The Glasgow monitoring of uninterrupted speech task (GMUST): A naturalistic measure of speech intelligibility in noise. In Proceedings of Meetings on Acoustics (Vol. 19, No. 1, p. 050068). Acoustical Society of America.

McGarrigle, R., Munro, K. J., Dawes, P., Stewart, A. J., Moore, D. R., Barry, J. G., \& Amitay, S. (2014). Listening effort and fatigue: what exactly are we measuring? A British Society of Audiology Cognition in Hearing Special Interest Group "white paper". International Journal of Audiology, 53(7), 433-440.

Moray, N. (1967). Where is capacity limited? A survey and a model. Acta Psychologica, 27, 84-92.

Pals, C., Sarampalis, A., \& Başkent, D. (2013). Listening effort with cochlear implant simulations. Journal of Speech, Language, and Hearing Research, 56(4), 1075-1084.

Pals, C., Sarampalis, A., van Rijn, H., \& Başkent, D. (2015). Validation of a simple response-time measure of listening effort. The Journal of the Acoustical Society of America, 138(3), EL187-EL192.

Piaget, J., \& Inhelder, B. (2008). The psychology of the child (H. Weaver, Trans.). New York: Basic Books. (Original work published 1966)

Pichora-Fuller, M. K., Kramer, S. E., Eckert, M. A., Edwards, B., Hornsby, B. W. Y., Humes, L. E., ... Wingfield, A. (2016). Hearing Impairment and Cognitive Energy: The Framework for Understanding Effortful Listening (FUEL). Ear and Hearing, 37(Suppl. 1), 5S-27S.

Picou, E. M., \& Ricketts, T. A. (2014a). Increasing motivation changes subjective reports of listening effort and choice of coping strategy. International Journal of Audiology, 53(6), 418-426.

Picou, E. M., \& Ricketts, T. A. (2014b). The effect of changing the secondary task in dual-task paradigms for measuring listening effort. Ear and Hearing, 35(6), 611-622.

Picou, E. M., Ricketts, T. A., \& Hornsby, B. W. (2013). How hearing aids, background noise, and visual cues influence objective listening effort. Ear and Hearing, 34(5), e52-e64.

Preisler, G., Tvingstedt, A.-L., \& Ahlström, M. (2005). Interviews with deaf children about their experiences using cochlear implants. American Annals of the Deaf, 150(3), 260-267.

Rönnberg, J., Lunner, T., Zekveld, A., Sörqvist, P., Danielsson, H., Lyxell, B., ... \& Rudner, M. (2013). The Ease of Language Understanding (ELU) model: theoretical, empirical, and clinical advances. Frontiers in Systems Neuroscience, 7, Article 31. 
Rudner, M., Karlsson, T., Gunnarsson, J., \& Rönnberg, J. (2013). Levels of processing and language modality specificity in working memory. Neuropsychologia, 51(4), 656-666.

Rudner, M., Lunner, T., Behrens, T., Thorén, E. S., \& Rönnberg, J. (2012). Working memory capacity may influence perceived effort during aided speech recognition in noise. Journal of the American Academy of Audiology, 23(8), 577-589.

Sarampalis, A., Kalluri, S., Edwards, B., \& Hafter, E. (2009). Objective measures of listening effort: Effects of background noise and noise reduction. Journal of Speech, Language, and Hearing Research, 52(5), 1230-1240.

Schaerlaekens, A. M., Kohnstamm, G. A., Lejaegere, M., \& Vries, A. K. (1999). Streeflijst woordenschat voor zesjarigen: gebaseerd op nieuw onderzoek in Nederland en België. Swets \& Zeitlinger.

van Wieringen, A., \& Wouters, J. (2015). What can we expect of normally-developing children implanted at a young age with respect to their auditory, linguistic and cognitive skills?. Hearing Research, $322,171-179$.

Venail, F., Vieu, A., Artieres, F., Mondain, M., \& Uziel, A. (2010). Educational and employment achievements in prelingually deaf children who receive cochlear implants. Archives of Otolaryngology-Head \& Neck Surgery, 136(4), 366-372.

Wålinder, R., Gunnarsson, K., Runeson, R., \& Smedje, G. (2007). Physiological and psychological stress reactions in relation to classroom noise. Scandinavian Journal of Work, Environment \& Health, 33(4), 260-266.

Zekveld, A. A., Kramer, S. E., \& Festen, J. M. (2010). Pupil response as an indication of effortful listening: The influence of sentence intelligibility. Ear and Hearing, 31(4), 480-490.

\section{Figure Legends}

Figure 1: In each trial of the behavioural paradigm developed for the present study, the participants were instructed to complete three tasks in a fixed order. (I) was the listening phase, where a target word was played (in quiet or in noise) while the child looked at a fixation cross on the response screen. At the end of the word playback, the screen changed to one of the three shown in phase (II) according to the categorization condition of the current run. During (II), the child had to touch one of the two buttons as quickly as possible to indicate his/her judgment with regards to the categorization question, and the software recorded the response time. Phase (III) followed, where the child repeated what he/she heard back to the tester, who then scored the verbal response. "Spion" is a Dutch noun meaning 'spy'.

Figure 2: Error bars denote +/- 1 SD. An overall ANOVA revealed a significant main effect of different signal-to-noise ratios (SNR) as well as a significant main effect of different categorization tasks (Task). There was also significant interaction between SNR and Task. Subsequent post hoc analysis revealed that the mean response time (RT) at each of the three SNR levels («Quiet», "0 SNR», and «-5 SNR») was significantly different from the mean RTs at the other two SNR levels. Moreover, that mean RT at each 
of the three Task levels ("Color», "Animal», and «Dangerous») was also significantly different from the mean RTs at the other two Task levels. 
Table 1: Percentage (\%) distribution of word syllable count in the four semantic categories

\begin{tabular}{|lcccc|}
\hline & $\begin{array}{c}\text { Harmless } \\
\text { Animals }\end{array}$ & $\begin{array}{c}\text { Harmless } \\
\text { Non-animals }\end{array}$ & $\begin{array}{c}\text { Dangerous } \\
\text { Animals }\end{array}$ & $\begin{array}{c}\text { Dangerous } \\
\text { Non-animals }\end{array}$ \\
\hline \% 1-syllable words & 46 & 56 & 50 & 44 \\
\% 2-syllable words & 41 & 31 & 30 & 40 \\
\% 3-syllable words & 13 & 13 & 15 & 12 \\
\% 4-syllable words & 0 & 0 & 5 & 4 \\
\hline
\end{tabular}

Table 1 Legend: Shown here is that the distribution of words of different syllable lengths is roughly even among the four distinct semantic categories. 
Table 2: Median Word Recognition Score (\%) in each condition (Interquartile Range in parenthesis)

\begin{tabular}{|c|c|c|c|}
\hline & Quiet & $0 \mathrm{SNR}$ & $-5 \mathrm{SNR}$ \\
\hline Color & $100(3)$ & $93(10)$ & $77(11)$ \\
\hline Animal & $100(3)$ & $93(7)$ & $73(10)$ \\
\hline \multirow[t]{3}{*}{ Dangerous } & $100(3)$ & $93(7)$ & $73(18)$ \\
\hline & $L$ & 11 & $\downarrow$ \\
\hline & & & \\
\hline
\end{tabular}

Table 2 Legend: *** $p<0.001$ in post hoc Wilcoxon signed-rank test, Bonferroni-corrected alpha $=0.017$. 
Table 3: Mean percentage (\%) of 'incorrect' categorization responses in each condition

\begin{tabular}{|cccc|}
\hline & Quiet & 0 SNR & -5 SNR \\
\hline Color & $0.6 / 0.0 / 0.6$ & $0.9 / 0.1 / 0.8$ & $1.3 / 1.2 / 0.1$ \\
Animal & $5.2 / 1.2 / 4.0$ & $6.7 / 3.7 / 3.0$ & $14.0 / 11.2 / 2.8$ \\
Dangerous & $15.6 / 0.7 / 14.9$ & $17.8 / 3.3 / 14.4$ & $22.7 / 12.6 / 10.1$ \\
\hline
\end{tabular}

Table 3 Legend: The first number in each condition represents the mean total percentage of trials where a child made an incorrect categorization judgment; the second number represents the mean percentage of those trials where both verbal repetition and categorization were incorrect; the third number represents the mean percentage of trials where a child repeated a word correctly but still made an incorrect categorization choice. 
Figure 1: Overview of the listening effort paradigm at trial level

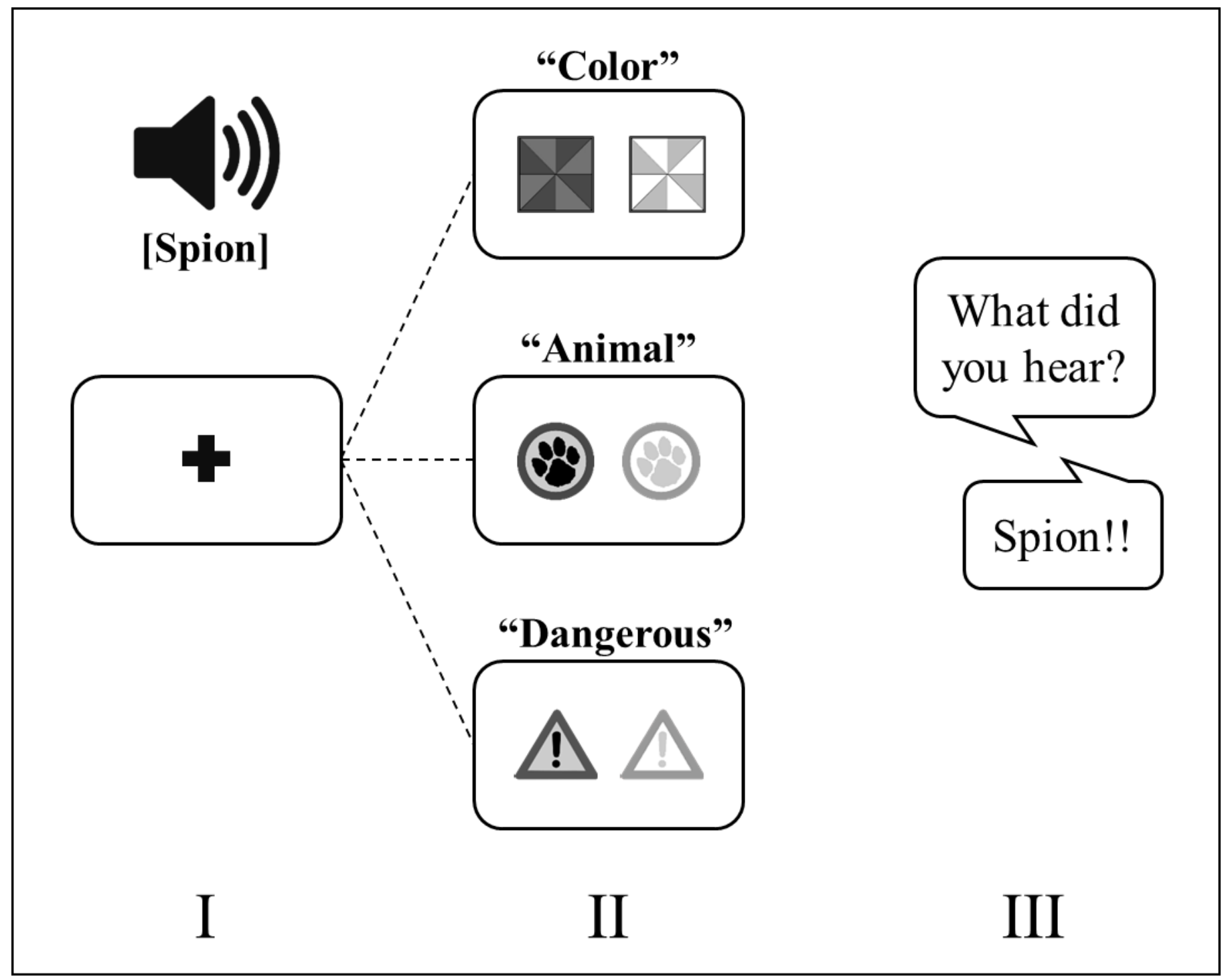


Figure 2: Mean Response Time results grouped by conditions

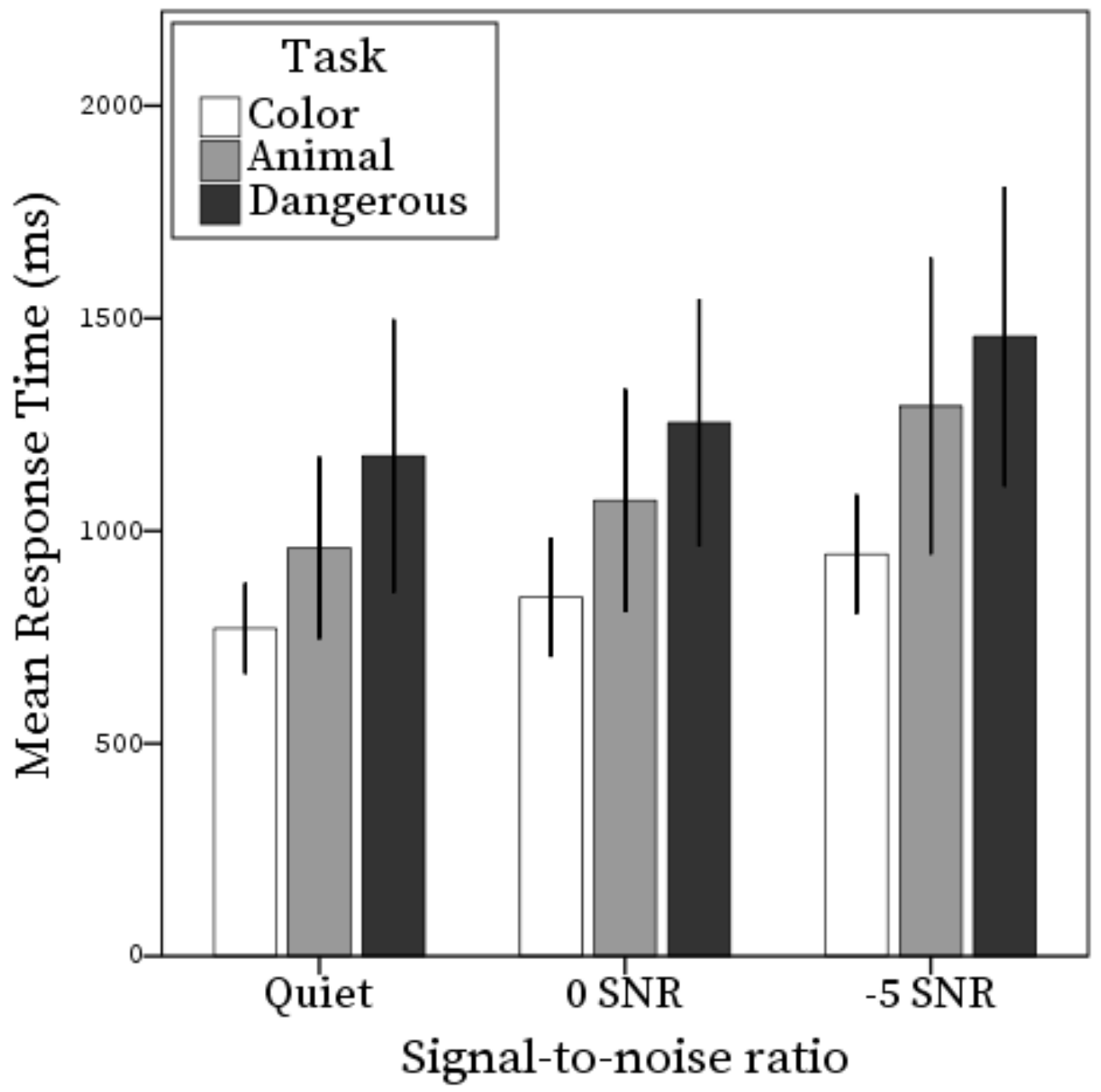

